\title{
Perceptual versus postperceptual mediation of visual context effects: Evidence from the letter-superiority effect
}

\author{
EYAL M. REINGOLD \\ University of Toronto, Mississauga, Ontario, Canada \\ and \\ PIERRE JOLICOEUR \\ University of Waterloo, Waterloo, Ontario, Canada
}

\begin{abstract}
Two experiments demonstrated letter-context effects that cannot easily be accounted for by postperceptual theories based on structural redundancy, figural goodness, or memory advantage. In Experiment 1, subjects identified the color of a letter fragment more accurately in letter than in nonletter contexts. In Experiment 2, subjects identified the feature presented in a precued color more accurately in letters than in nonletters. We argue that these effects result from topdown perceptual processing.
\end{abstract}

Briefly presented line segments are identified more accurately when they are embedded in coherent object-like contexts than when they are either embedded in noncoherent contexts or presented alone. These visual-context effects are referred to as the object-superiority effect (OSE) and the object-line effect (OLE), respectively. The original demonstrations of the OSE (Weisstein \& Harris, 1974) and the OLE (Williams \& Weisstein, 1978) were aimed at drawing an "analogy between object and word perception, and opening further opportunities for studying the effects of contexts on the perception of constituent elements without being restricted to linguistic material" (Williams \& Weisstein, 1978, p. 85). Similarly, the welldocumented context effects in word perception inspired the exploration of a variety of visual-context effects with stimuli such as faces (e.g., Gyoba, Arimura, \& Maruyama, 1980; Homa, Haver, \& Schwartz, 1976; Purcell \& Stewart, 1986, 1988; van Santen \& Jonides, 1978), letters (Schendel \& Shaw, 1976), and real-world scenes (Biederman, 1972, 1981; Biederman, Glass, \& Stacey, 1973; Palmer, 1975).

This research was supported by NSERC Grant A2579 to P. J. and NSERC Grant OGP0105451 to E. M. R. We are indebted to Liz Bosman, Phil Merikle, and Derek Besner for many constructive discussions of the issues, to Keith McGowan for writing the software, and to Susan Fraser for assisting in data collection. We would especially like to acknowledge Lester Krueger's helpful input during the process of revising earlier drafts of this paper. We also thank James Enns and two anonymous reviewers for their contributions. Correspondence should be addressed to either E. M. Reingold, Department of Psychology, University of Toronto, Erindale College, 3359 Mississauga Road, Mississauga, Ontario, Canada LSL 1C6 (e-mail: reingold@psych.toronto.edu); after July 1993: School of Psychology, The University of New South Wales, P.O. Box 1, Kensington, New South Wales, 2033, Australia; or P. Jolicoeur, Department of Psychology, University of Waterloo, Waterloo, Ontario, Canada N2L 3G1.
Despite the intriguing surface similarities between the various visual-context effects, it may be premature to conclude that these effects are, in fact, comparable. For example, Weisstein and her colleagues (e.g., Weisstein \& Harris, 1974; Williams \& Weisstein, 1978) viewed the OSE and the OLE as analogous to the word-superiority effect (WSE) and the word-letter effect (WLE), respectively (for reviews of the WSE literature, see Baron, 1978; Johnston, 1981; Krueger, 1975). However, this position has been challenged recently by Enns and Prinzmetal (1984). These investigators argued "that the objectsuperiority and object-line effects are not strictly analogous to the word and scene effects to which they are often compared" (p. 26). More specifically, Enns and Prinzmetal claimed that while the WSE truly reflects subjects' preexperimental familiarity with the stimuli (i.e., words), in their experiments "there was no evidence of observers using a priori knowledge of structural relations in the stimuli to facilitate target identification. They seemed to rely only on redundancy they could detect in the stimulus set to facilitate their performance"' (p. 26). Enns and Prinzmetal's position is based on a redundancy account of the OSE and OLE. It is important to note that the type of model proposed by Enns and Prinzmetal is not without precedent in the history of the study of visual-context effects. As pointed out by Baron (1978), the WSE "history can be understood largely in terms of a series of efforts to rule out explanations of the effect that would reduce it to some other well-known phenomenon, such as the use of informational redundancy in guessing" (p. 131). Indeed, the process of ruling out redundancy theories of the WSE required highly sophisticated and carefully controlled research, as exemplified by the seminal work of Johnston $(1974,1978$; for reviews, see Baron, 1978; Johnston, 1981). The suggestions of Enns and 
Prinzmetal may serve as a reminder that researchers of context effects in object perception face a similar challenge.

The main purpose of the present paper is to suggest a paradigm for the study of visual-context effects in object perception that will provide the necessary controls for ruling out explanations based upon the concept of redundancy. In particular, we were interested in studying context effects in the perception of highly familiar objects. Accordingly, we first discuss issues related to the role of redundancy in object perception. Next, we outline the rationale of the present approach. Finally, we illustrate the proposed approach by applying it to the study of visualcontext effects in the perception of a specific class of highly familiar objects, namely letters.

\section{Redundancy and Context Effects in Object Perception}

The concept of redundancy may be relevant to the study of visual-context effects in at least three different ways:

1. Structural redundancy within the experimental stimulus set. Subsequent to the initial demonstrations of the OSE and OLE by Weisstein and her colleagues, most of the research in this area has been devoted to the exploration of the boundary conditions of these effects (Chen, 1982; Earhard, 1980; Earhard \& Armitage, 1980; Klein, 1978; Lanze, Maguire, \& Weisstein, 1985; Lanze, Weisstein, \& Harris, 1982; McClelland, 1978; McClelland \& Miller, 1979; Pomerantz, 1981; Pomerantz, Sager, \& Stoever, 1977; Weisstein, Williams, \& Harris, 1982; Womersley, 1977). Most researchers studying the OSE and OLE have implicitly assumed zero redundancy between the contexts and the target lines within the stimulus sets employed in their experiments. In other words, contexts were assumed to be noninformative, or nonconstraining, and therefore could not be used by subjects to predict or deduce the identity of the target lines. This was assumed because given any of $N$ contexts, $C_{1}, C_{2}, \ldots C_{i}$, $\ldots C_{n}$, or the null context, $C_{\mathrm{o}}$ (i.e., line alone), the alternative target lines, for example, $A$ and $B$, had equal probability of occurrence, that is, $P\left(A \mid C_{i}\right)=P\left(B \mid C_{i}\right)$. However, as correctly pointed out by Enns and Prinzmetal (1984), target lines may interact with context lines to produce higher order local or global forms such as angles or bounded regions, which in turn may be correlated with target lines resulting in greater than zero redundancy. If, for example, the interaction between Line $A$ and each one of the contexts, $\left[C_{i} \& A\right]$, always produces an angle in the shape of an arrow, whereas the interaction of Line $B$ with the same contexts, $\left[C_{i} \& B\right]$, always produces a bounded region in the shape of a triangle, then there is perfect redundancy (i.e., a correlation of 1 ) between the higher order forms arrow and triangle, and the Target Lines $A$ and $B$.

Enns and Prinzmetal (1984) manipulated the magnitude of the redundancy in separate stimulus sets by varying the degree to which the shapes of a triangle and arrow were correlated with the Target Lines $A$ and $B$. The re- sults of this experiment indicated that, across stimulus sets, line-in-context identification accuracy was positively correlated with the magnitude of the structural redundancy. In fact, contexts (i.e., triangle or arrow) facilitated lineidentification performance only under conditions of abovezero structural redundancy within the stimulus set.

Although not all of the findings reported by researchers of the OSE and OLE can be explained solely in terms of structural redundancy within the experimental stimulus set (see Enns \& Gilani, 1988; Lanze et al., 1985), the findings reported by Enns and Prinzmetal (1984) clearly demonstrate the potential relevance of this factor to the study of context effects in object perception.

2. Preexperimental knowledge of structural redundancies. Enns and Prinzmetal (1984) emphasized the importance of subjects' ability to be informed and influenced by the structural redundancy within the stimulus set during the experimental session. However, when studying visual-context effects with familiar everyday objects, an additional source for subjects' knowledge of structural redundancies, namely preexperimental knowledge, needs to be considered. As pointed out by Kinchla (1977), subjects may employ their knowledge of a correlation between familiar visual images and their parts (i.e., structural redundancy) to infer, rather than actually "see," those parts when the objects are presented for a very brief duration. In fact, familiar everyday objects are highly redundant. For any given object, some features, parts, or attributes are more likely than others, and similarly, some spatial arrangements of these attributes are more likely than others. Thus, familiar object contexts are more constraining or informative (i.e., redundant) relative to nonobject contexts (i.e., unfamiliar arrangements of object parts).

The above considerations present a fundamental theoretical problem in the interpretation of visual-context effects in the perception of familiar objects (i.e., superior identification of component parts in object, relative to nonobject, contexts). More specifically, subjects' knowledge of the structural redundancy in objects may enable them to supplement an incomplete perceptual analysis (i.e., a fragmentary percept) of an object by inferring, or guessing, the missing component part(s). In contrast, familiarity with objects may alter the perceptual processing of attributes such that these attributes are more perceptible when embedded in object contexts relative to nonobject contexts. The former is a postperceptual interpretation of visual-context effects commonly referred to as the redundancy theory or the sophisticated-guessing hypothesis (see Johnston, 1978, 1981), whereas the latter is a top-down perceptual-processing interpretation of this phenomenon (see McClelland \& Rumelhart, 1981).

As an illustration of the two opposing interpretations of visual-context effects described above, consider the following accounts of the WSE. According to a redundancy, or sophisticated-guessing, account of the WSE, knowledge of the structural redundancy in the English language restricts, or constrains, the alternative completions of a 
fragmentary percept of a word (e.g., given CLU_, only " $B$ " or " $E$ " are possible, and " $B$ " is more likely than

" $E$ "). Consequently, word contexts, but not nonword contexts (i.e., random-letter strings), allow subjects to make informed decisions as to the identity of letters that were not fully processed, resulting in a superior letteridentification performance in the former relative to the latter condition (i.e., WSE). In contrast, the interactiveaclivation model proposed by McClelland and Rumelhart (1981) provides a lop-down perceptual-processing account of the WSE. According to this model, activation from letter-level representations excites consistent word-level representations, which in tum feed back to the letter level, strengthening the activation of constituent letters and thereby increasing their perceptibility. Thus, according to the interactive-activation model, letters in words are actually "seen" better than letters in nonwords.

Note that within the framework of the interactiveactivation model, subjects' knowledge of the structural redundancy in the English language is implicitly embodied in the specific representations of words. Consequently. top-down perceptual-processing accounts of visual-context effects such as the interactive-activation model technically may also be viewed as redundancy theories. However, traditionally, the term redundancy is reserved for models that postulate that structural redundancy influences postperceptual decision processes.

As mentioned earlier, the general consensus among researchers of the WSE is that redundancy theories have been ruled out as a potential explanation for that phenomenon (see Baron, 1978; Johnston, 1981; McClelland \& Rumelhart, 1981). However, to date, redundancy models have not been extensively evaluated in the study of visualcontext effects in object perception. Several unresolved issues make it difficult to apply research strategies that were developed in the study of context effects in word perception to similar investigations in the object domain. First, the segmentation of an object into its component parts is often far more complicated than the segmentation of a word into its constituent letters (see Pomerantz, 1981). Second, a somewhat related problem is that a method for quantifying the level of contextual constraint (i.e., structural redundancy) for a given component part of a familiar object is not readily available (see Kinchla, 1977). Thus, it seems that a successful evaluation of the relationship between visual-context effects and redundancy in object perception requires the development of new experimental paradigms.

3. Figural goodness. Finally, there is another aspect of stimulus redundancy that may prove to be relevant to the study of context effects in object perception. This aspect is traditionally referred to as figural goodness. The notion of figural goodness originated from the approach of Gestalt psychology to pattem recognition. The introduction of information-theory concepts in psychology provided valuable tools for quantifying figural goodness (for reviews, see Attneave, 1959; Gamer, 1962, 1974). In particular, the concept of informational redundancy proved to be useful for that purpose.
The most extensively researched redundancy index of figural goodness is Garner's (1962, 1974) rotation and reflection (R\&R) measure. The $R \& R$ value for any given stimulus equals the number of nonequivalent patterns produced by its rotation and reflection. Gamer and Clement (1963) showed that the R\&R index correlates highly with the way subjects rate goodness. As pointed out by Attneave (1959), the aspect of figural goodness best captured by redundancy indexes is symmetry.

The potential importance of figural goodness to the study of visual-context effects in object perception stems from the fact that many familiar natural objects and manmade objects and symbols tend to be good patterns and are often symmetric. Thus, depending on the objects and nonobjects employed in an experiment demonstrating a context-superiority effect, figural goodness, rather than familiarity, may account for that effect.

To summarize, structural redundancy provides a very powerful framework for explaining visual-context effects in object perception. There are two potential sources for subjects' knowledge of structural redundancies, preexperimental knowledge (i.e., stimulus familiarity) and experimentally learned knowledge (as proposed by Enns and Prinzmetal, 1984). Finally, adequate controls for both siructural redundancy and figural goodness are required if conclusive evidence against redundancy theories of context effects in object perception is to be obtained. Before outlining the controls for the altemative explanations of structural redundancy and figural goodness provided by the present approach, it is instructive to review briefly a different solution to the same problems, which was implemented in the snudy of the face-superionity effect (FSE) (e.g.. Gyoba et al., 1980; Homa et al., 1976; van Santen \& Jonides, 1978).

\section{The Face-Superiority Effect}

The FSE paradigm handles the issue of structural redundancy by employing several exemplars of each feature (i.e., eyes, nose, mouth, etc.). Different combinations of these features are used to produce faces and scrambled faces with a zero correlation between feature types, or exemplars, and stimulus types (i.e., faces and scrambled faces). After the stimulus presentation, subjects are required to recognize which one of the exemplars of a particular feature was shown.

Under these conditions, knowledge of the structural redundancies in the human face is rendered noninformative. This is the case because subjects" perception that a face was presented may allow them to infer that a particular feature, for example, a nose, had been presented, but not which type of nose it was. Furthermore, the reasonably large number of contexts typically employed in FSE experiments (e.g., van Santen \& Jonides, 1978, employed 16 faces and 16 scrambled faces with only one repetition of each stimulus) makes it highly unlikely that subjects learn to recognize specific faces and therefore establish stnuctural redundancies during the experimental session. In addition, in the original demonstration of the FSE by Homa et al. (1976), the effect of figural good- 
ness was controlled by employing symmetric scrambled faces. Given the adequate controls employed in the FSE paradigm, the documented context effects obtained within that paradigm cannot be explained by a redundancy model and in that respect may be considered analogous to the WSE.

However, there is a limited range of objects for which an approach like the one employed in the study of the FSE is applicable. This is the case because an FSE-like paradigm requires employing stimuli that share a common prototype. Nevertheless, testing the same approach with other classes of similar stimuli such as chairs as well as mixing objects of more than one type represent very interesting possible extensions of the FSE.

\section{The Proposed Paradigm}

The control proposed by the present paradigm for the potential influence of structural redundancy is based on dissociating any form characteristics from the stimulus dimension to which subjects are required to respond. The stimuli used in the paradigm vary along two dimensions, form and color. The color dimension was selected because it is generally considered as separable, or independent from the dimension of form (e.g., Gottwald \& Garner, 1972; Handel \& Imai, 1972; Nissen, 1979; Prinzmetal, Presti, \& Posner, 1986; Treisman \& Gelade, 1980; Treisman \& Schmidt, 1982). Each one of the features of the stimuli (i.e., objects and matched nonobjects) would be equally likely to appear in each one of the alternative colors, that is, there would be no correlation between the form and color dimensions. On any given trial, subjects would be required to judge the color of a certain feature in the stimulus that was presented.

As in the FSE paradigm, any knowledge subjects may have about the structural redundancies in the objects included in the stimulus set would be rendered noninformative. This is the case because identifying the presented object may enable subjects to infer the presentation of its parts, but not their color. This argument applies just as well to any structural redundancies that may be learned during the experimental sessions via the availability of diagnostic emergent features such as the ones proposed by Enns and Prinzmetal (1984).

The suggested paradigm allows for several possible controls necessary for disentangling familiarity and figural goodness. Whenever possible, objects and nonobjects would be equated with respect to figural goodness. Given that this solution may not always be possible, the effect of figural goodness can be tested by employing two baselines: nonobjects with high figural goodness and nonobjects with low figural goodness. Another potentially useful control stimulus set can be produced by using nonequivalent rotations or reflections of the objects employed in the experiments, which, according to Garner's R\&R index, are by definition equally good patterns.

\section{Application of the Proposed Approach to the Study of Context Effects in the Perception of Letters}

For three reasons, we chose to illustrate the proposed paradigm by applying it to the study of visual-context effects in the perception of letters. First, letters can be parsed into fragments (i.e., line segments) more easily than most familiar objects. Second, letters typically appear in a monochromatic color, thus making it safe to assume that color is an irrelevant dimension in the perceptual processing of letters. In other words, there are no natural regularities associating specific features of letters with certain colors. Third, context effects in the perception of letters have not been extensively researched. To date, we know of only one report documenting such an investigation (Schendel \& Shaw, 1976).

Schendel and Shaw (1976) attempted to test the generality of the finding that briefly presented letters are identified more accurately when embedded in words than when presented alone (i.e., the WLE; e.g., Reicher, 1969). The stimuli employed in their experiments were letter pairs such as $\mathrm{H}$ and $\mathrm{N}$, as well as the critical letter fragments that distinguished between the members of each pair (e.g., - and $\backslash$ for $\mathrm{H}$ vs. $\mathrm{N}$ ). Eight letter pairs and their corresponding eight critical fragment pairs were used. In Experiment 1 , following the stimulus presentation of a letter or a fragment, subjects attempted to recognize which one of the pair of relevant letter fragments was presented. Under these conditions, it was found that fragments were identified faster and more accurately when presented in the context of a letter than when presented alone. Schendel and Shaw referred to this finding as the letter-context effect (LCE).

However, the LCE can easily be accounted for in terms of a structural-redundancy explanation. Subjects may use their knowledge of the structural redundancies in letters, that is, the correlation between letters and their fragments, in improving identification performance in the fragmentin-context condition, whereas such information is not available in the fragment-alone condition. In fact, this is very similar to the interpretation of the LCE adopted by Schendel and Shaw (1976). They derived additional support for that explanation from the results of their Experiment 3 . In this experiment, the pair of fragment alternatives were presented prior to the stimulus presentation. Under these conditions, fragment identification was at least as good in the fragment-alone condition as in the fragment-in-context condition. These results are consistent with a redundancy model of the LCE. This is the case because when prior knowledge of the possible target alternatives was available, the letter-in-context condition was no longer more informative or constraining than the fragment-alone condition (see Massaro, 1973).

In the present experiments, we attempted to demonstrate a context effect in letter perception that cannot be explained by a redundancy model. Experiment 1 demon- 
strated such an effect, and Experiment 2 provided convergent evidence, as well as a preliminary indication suggesting that the locus of this effect may be in perception rather than memory.

\section{EXPERIMENT 1}

Experiment 1 was designed to explore the influence of letter contexts on the perception of constituent elements (i.e., letter features) under conditions that provided adequate controls for the effects of structural redundancy and figural goodness. The stimulus types used in the experiment were letters and two matched sets of nonletters that were produced by combining features from different letters. Figural goodness was manipulated across the two nonletter sets (i.e., high goodness vs. low goodness) to provide the necessary controls for disentangling its effects from the effects of stimulus familiarity (i.e., letters vs. nonletters).

On each trial following the presentation of a stimulus (i.e., a letter or a nonletter) and a backward feature mask, one of the features of the stimulus was presented. Subjects were required to judge in which of the two alternative colors, red or green, this feature was displayed in the stimulus.

For each stimulus, color and form were uncorrelated, that is, each one of its features was equally likely to appear in each one of the color alternatives employed. Thus, in the present experiment, subjects' knowledge of the structural redundancies in letters was rendered noninformative because the perception of any given letter could only have been used to infer its features, not their color.

Finally, the nonletter sets were carefully matched to the letter sets in terms of their features and in terms of local and global form characteristics. Thus, it was postulated that any letter-context-superiority effect obtained within the present paradigm would not be attributable to the influence of either structural redundancy or figural goodness.

\section{Method}

Subjects. Twenty undergraduate students at the University of Waterloo participated in the experiment. All subjects had normal or corrected-to-normal vision, and each subject was paid $\$ 10$ following completion of the experimental session.

Apparatus. All stimulus materials were displayed on the color monitor of an Amiga 1000 microcomputer. All stimuli were presented binocularly, and a chinrest maintained viewing distance at $90 \mathrm{~cm}$. The experiment was run in a lighted room where the luminance was approximately $30 \mathrm{~cd} / \mathrm{m}^{2}$.

A mouse with two buttons was used as an input device. The mouse was located on a table in front of the subjects. The mouse was positioned in a manner that allowed the subjects' hands to rest comfortably on the table. The right mouse button was used to initiate trials, and the left buttion was used to indicate a decision following each presentation sequence.

Materials and Design. As a first step in the construction of the stimulus set used in the experiment, we selected the uppercase letters A, B, F, H, I, K, N, R, Y, and Z. These 10 letters were selected because they could easily be parsed into three fragments, or features. Over the 10 letters, there were 21 nonequivalent features. For our purpose, two features were considered nonequiva- lent even when they differed only in terms of their orientation or location (e.g., the two vertical line segments in an $\mathrm{H}$ ). Some features occurred more than once within the letter set (e.g., the leftmost vertical line in $\mathrm{B}, \mathrm{F}, \mathrm{H}, \mathrm{K}, \mathrm{N}$, and $\mathrm{R}$ ), accounting for the existence of 21 rather than 30 nonequivalent features. The features used in constructing the letter set are presented in Figure 1.

The features of the letter set were combined to produce two nonletter sets: a high-figural-goodness nonletter set and a low-figuralgoodness nonletter set. The mean $R \& R$ values for the letters, highfigural-goodness nonletters, and low-figural-goodness nonletters were $4.4,5.2$, and 8 , respectively. To compute the $R \& R$ value, each one of the 30 stimuli was rotated in $90^{\circ}$ steps. Each one of the four resulting patterns was then reflected about the vertical axis, producing a total of eight patterns. The R\&R value for any given stimulus equaled the number of nonequivalent patterns generated by this procedure. Thus, the possible range of $R \& R$ values is $1-8$, with a lower score indicating a higher level of figural goodness. In addition, familiarity ratings for each of the letters and nonletters were obtained. Fifty subjects rated each stimulus on a scale from 0 (very unfamiliar) to 10 (very familiar). The 30 stimuli were randomly intermixed during the collection of the familiarity ratings. The letters, high-figural-goodness nonletters, and low-figuralgoodness nonletters are presented in Figures 2, 3, and 4, respectively.

The same features with the same frequency of occurrence were used in producing the letters and each of the two nonletter sets. As a result, each feature appeared in identical orientation and spa-

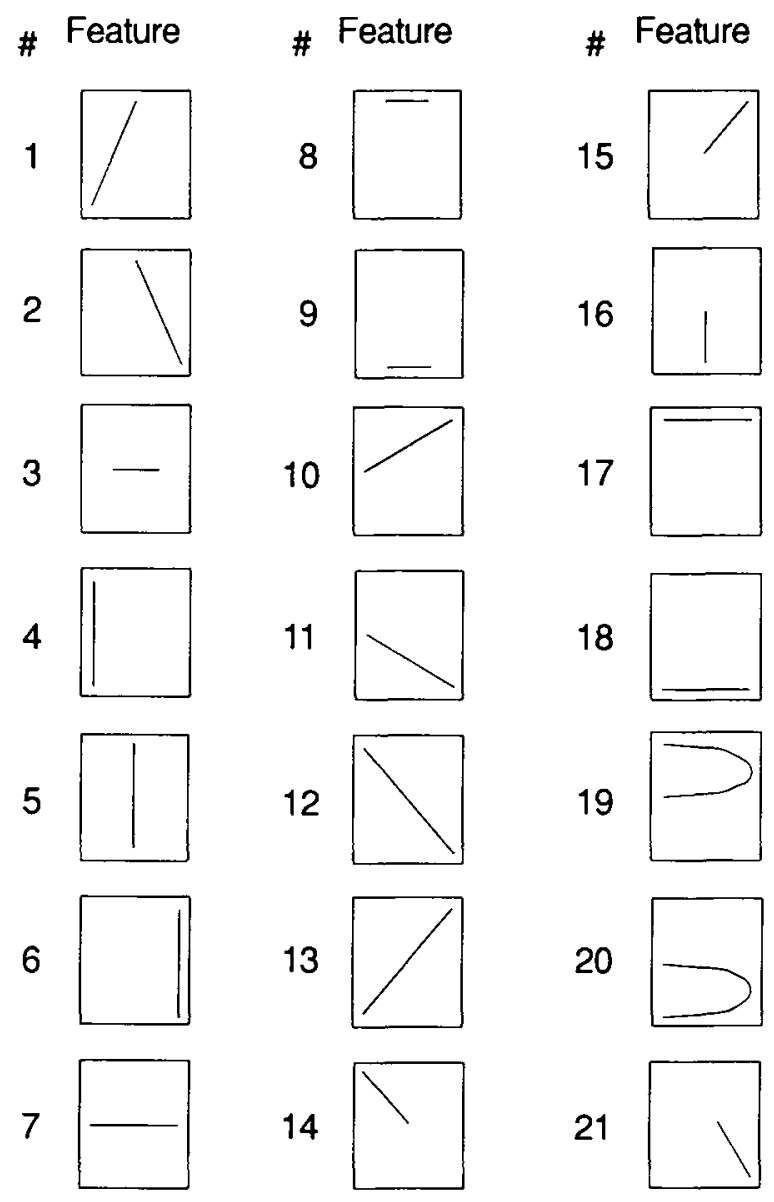

Figure 1. Features used in constructing the stimulus sets in Experiments 1 and 2. 


\begin{tabular}{|c|c|c|c|c|c|}
\hline \multirow{2}{*}{ Stinulus } & \multirow{2}{*}{ Features } & \multirow{2}{*}{$\begin{array}{l}\text { RRR } \\
\text { Value }\end{array}$} & \multirow{2}{*}{$\begin{array}{l}\text { Mean } \\
\text { Familiarity } \\
\text { Ratings }\end{array}$} & \multicolumn{2}{|c|}{ Proportion Comect } \\
\hline & & & & Exp. 1 & Exp. 2 \\
\hline & $1,2,3$ & 4 & 10.00 & .742 & .750 \\
\hline & $4,19,20$ & 4 & 9.71 & .833 & .814 \\
\hline & $4,7,17$ & 8 & 9.91 & .797 & .839 \\
\hline & $4,6,7$ & 2 & 9.96 & .781 & .858 \\
\hline & $5,8,9$ & 2 & 9.86 & .761 & .769 \\
\hline & $4,10,11$ & 4 & 9.89 & .791 & .867 \\
\hline & $4,6,12$ & 4 & 9.96 & .853 & .875 \\
\hline & $4,19,21$ & 8 & 9.93 & .822 & .814 \\
\hline & $14,15,16$ & 4 & 9.96 & .814 & .839 \\
\hline & $13,17,18$ & 4 & 9.84 & .805 & .794 \\
\hline & & 4.4 & 9.90 & .800 & .822 \\
\hline
\end{tabular}

Figure 2. The feature composition, $R \& R$ values, mean familiarity ratings, and mean proportion correct for the letters used in Experiments 1 and 2 . The constituent features are specified by their corresponding numbers in Figure 1.

tial location in each set. Also, we attempted to match stimuli across sets in terms of size, complexity, connectedness, and structural relevance of features. Furthermore, all the stimuli employed in the experiment were perceived as two-dimensional patterns.

In addition to the stimuli used in the experimental trials, four different stimuli were used during the practice trials. These stimuli were two numbers, 4 and 5 , and two nonnumbers that were constructed in a manner similar to the letter and nonletter sets. That is, the numbers and the non-numbers represented different combinations of exactly the same features in the same spatial location.

Stimulus presentation was always followed by a mask, which was constructed by overlapping all the features used in producing the experimental and practice stimuli. The experimental and practice stimuli, as well as the mask, were identical in size, measuring $3 \mathrm{~cm}$ $\left(1.9^{\circ}\right)$ horizontal by $4 \mathrm{~cm}\left(2.6^{\circ}\right)$ vertical. In addition, a fixation frame measuring $3.6 \mathrm{~cm}\left(2.3^{\circ}\right)$ horizontal by $4.6 \mathrm{~cm}\left(2.9^{\circ}\right)$ vertical was always presented during the trial sequence.

On any trial, each of the three features of the stimulus was equally likely to appear in red or green. Two of the features appeared in one of these colors, and the third appeared in the other color. Hence, for each stimulus, there were six possible feature-color mappings. The mask and the fixation frame were presented in white, and the background color was black. The red and green colors were similar in brightness and saturation.

A total of 540 experimental trials were generated by using all possible combinations of 30 stimuli $\times 6$ feature-color mappings $\times 3$ potential target features. The experimental trials were divided into 18 blocks of 30 trials. Each block contained a single presentation of each of the 30 stimuli; no more than four consecutive trials displayed the target feature in the same color. Within these constraints, the assignment of trials to blocks and the presentation order of trials within each block were random. A different random order was generated for each subject.

The practice trials were composed of the 72 possible combinations of 4 stimuli $\times 6$ feature-color mappings $\times 3$ potential target features. These 72 practice trials were divided into 18 blocks of four trials, with a single presentation of each stimulus in a block. No more than four consecutive trials displayed the target feature in the same color. Within these constraints, the assignment of trials to blocks and the presentation order of trials within each block were random. A different random order was generated for each subject. Two such sets of 72 trials were administered consecutively to each subject, for a total of 144 practice trials.

\begin{tabular}{|c|c|c|c|c|c|}
\hline \multirow{2}{*}{ Stimulus } & \multirow{2}{*}{ Featumes } & \multirow{2}{*}{$\begin{array}{l}\text { Rer } \\
\text { Valuo }\end{array}$} & \multirow{2}{*}{$\begin{array}{c}\text { Moan } \\
\text { Familiauity } \\
\text { Ratings }\end{array}$} & \multicolumn{2}{|c|}{ Proportion Comect } \\
\hline & & & & Exp. 1 & Exp. 2 \\
\hline & $1,2,8$ & 4 & 4.77 & .755 & .814 \\
\hline & $4,12,13$ & 4 & 4.91 & .753 & .800 \\
\hline & $3,5,9$ & 4 & 3.39 & .675 & .686 \\
\hline & $4,17,18$ & 4 & 8.41 & .830 & .806 \\
\hline & $7,10,11$ & 4 & 6.89 & .722 & .772 \\
\hline & $6,19,20$ & 4 & 3.09 & .753 & .772 \\
\hline & $4,6,17$ & 4 & 6.39 & .836 & .867 \\
\hline & $4,7,21$ & 8 & 4.07 & .761 & .747 \\
\hline & $4,14,16$ & 8 & 2.41 & .758 & .786 \\
\hline & $4,15,19$ & 8 & 4.96 & .761 & .753 \\
\hline & & 5.2 & 4.93 & .760 & .780 \\
\hline
\end{tabular}

Figure 3. The feature composition, R\&R values, mean familiarity ratings, and mean proportion correct for the high-figuralgoodness nonletters used in Experiments 1 and 2. The constituent features are specified by their corresponding numbers in Figure 1. 


\begin{tabular}{|c|c|c|c|c|c|}
\hline \multirow{2}{*}{ Stimulus } & \multirow{2}{*}{ Features } & \multirow{2}{*}{$\begin{array}{l}\text { R\&R } \\
\text { Yalue } \\
\end{array}$} & \multirow{2}{*}{\begin{tabular}{|c|} 
Mean \\
Familiarity \\
Ratings
\end{tabular}} & \multicolumn{2}{|c|}{ Proportion Correct } \\
\hline & & & & Exp. 1 & Exp. 2 \\
\hline & $4,12,15$ & 8 & 2.84 & .767 & .794 \\
\hline & $3,4,14$ & 8 & 1.86 & .767 & .794 \\
\hline & $4,7,10$ & 8 & 3.18 & .753 & .855 \\
\hline & $6,11,19$ & 8 & 0.66 & .750 & .778 \\
\hline & $4,18,21$ & 8 & 2.41 & .781 & .792 \\
\hline & $6,17,19$ & 8 & 1.55 & .697 & .717 \\
\hline & $5,9,13$ & 8 & 3.05 & .725 & .692 \\
\hline & $2,4,17$ & 8 & 1.84 & .811 & .847 \\
\hline & $4,7,16$ & 8 & 3.52 & .767 & .761 \\
\hline & $1,8,20$ & 8 & 1.68 & .717 & .781 \\
\hline I & & 8 & 2.26 & .754 & .776 \\
\hline
\end{tabular}

Figure 4. The feature composition, R\&R values, mean familiarity ratings, and mean proportion correct for the low-figural-goodness nonletters used in Experiments 1 and 2. The constituent features are specified by their corresponding numbers in Figure 1.

Procedure. Each subject was tested individually during a single session that lasted approximately $75 \mathrm{~min}$. A summary of the trial sequence is presented in the top half of Figure 5. Throughout the experiment, the fixation frame was always present in the visual field. The subjects were instructed to fixate the area outlined by this frame before initiating each trial by pressing the right button on the mouse. Once a trial was initiated, $183 \mathrm{msec}$ elapsed before the onset of the colored stimulus. The offset of the stimulus display was immediately followed by a white feature mask presented for $100 \mathrm{msec}$. Following the mask, one of the three features of the stimulus was presented in white. The poststimulus display also included red and green rectangular color patches that were positioned directly below the fixation frame. The subjects were required to judge in which color the target feature had been displayed in the stimulus. The subjects indicated their decision by moving the mouse so as to direct a cursor in the form of an arrow to the red or green rectangular color patch on the screen. Once the arrow was appropriately positioned, the subjects pressed the left button on the mouse to record their response.

Practice trials were used for two reasons. First, they familiarized the subjects with the task. Second, during the practice trials, the stimulus duration was gradually decreased from its initial value of $333 \mathrm{msec}$. On the basis of the practice-trial performance, each sub- ject was assigned a fixed stimulus-duration value expected to produce approximately $75 \%$ accuracy during the experimental trials. Sixteen subjects were assigned a stimulus duration of $50 \mathrm{msec}$, and the remaining 4 subjects were assigned a stimulus duration of $67 \mathrm{msec}$. For each subject, stimulus duration was constant throughout the experimental trials.

\section{Results and Discussion}

For each subject, mean proportion correct was computed for each of the three stimulus types (i.e., letters vs. high-figural-goodness nonletters vs. low-figural-goodness nonletters). A summary of mean proportion correct by stimulus type is presented in Table 1. Stimulus type had a significant effect on color-identification performance $[\mathrm{F}(2,38)=14.05, p<.001]$. Planned comparisons indicated that color identification was more accurate in letter contexts than in both high-figural-goodness nonletter contexts $[t(19)=4.22, p<.001]$ and low-figuralgoodness nonletter contexts $[t(19)=5.10, p<.001]$. There was no significant difference in performance across the two nonletter sets $[t(19)=.72, p=.48]$.

However, the subject analysis described above was not ideal for disentangling the effects of familiarity versus figural goodness or for quantifying the amount of variance predicted by these variables. This was the case because to equate the nonletter sets in terms of features, we had to include some nonletters with lower figural goodness in the high-figural-goodness set. In addition, the two nonletter sets differed from the letter set, as well as from each other, in terms of both familiarity and mean R\&R values. The item analysis described below was designed to address these issues.

Item analysis. Mean proportion correct, $R \& R$ values, and mean familiarity ratings for each of the stimuli employed in the experiment are shown in Figures 2, 3, and 4. It is important to note that unlike the stimulus sets (i.e., letters, high-figural-goodness nonletters, and low-figuralgoodness nonletters), which were identical in terms of their feature composition, any single item represented a unique combination of three features. Consequently, differences in color-identification performance across items may reflect item-level effects (i.e., context effects) as well as feature-level effects (i.e., differences in constituent fragments). Accordingly, adjusted performance scores were computed for each item by removing feature variance. As a first step, mean proportion correct for each of the 21 features used in constructing the stimulus sets (see Figure 1) was computed. If item performance was determined solely by its feature composition, then it would be expected to equal the average performance across its constituent features. Accordingly, obtained itemperformance values were residualized with respect to performance values predicted by item-feature composition. The residuals from this analysis represent the part of itemperformance variance that is not predictable on the basis of feature performance. These residuals are henceforth referred to as adjusted item-performance scores.

Table 2 shows the correlations between familiarity ratings and figural goodness (i.e., R\&R values) and item performance. Table 2 includes correlations with item per- 


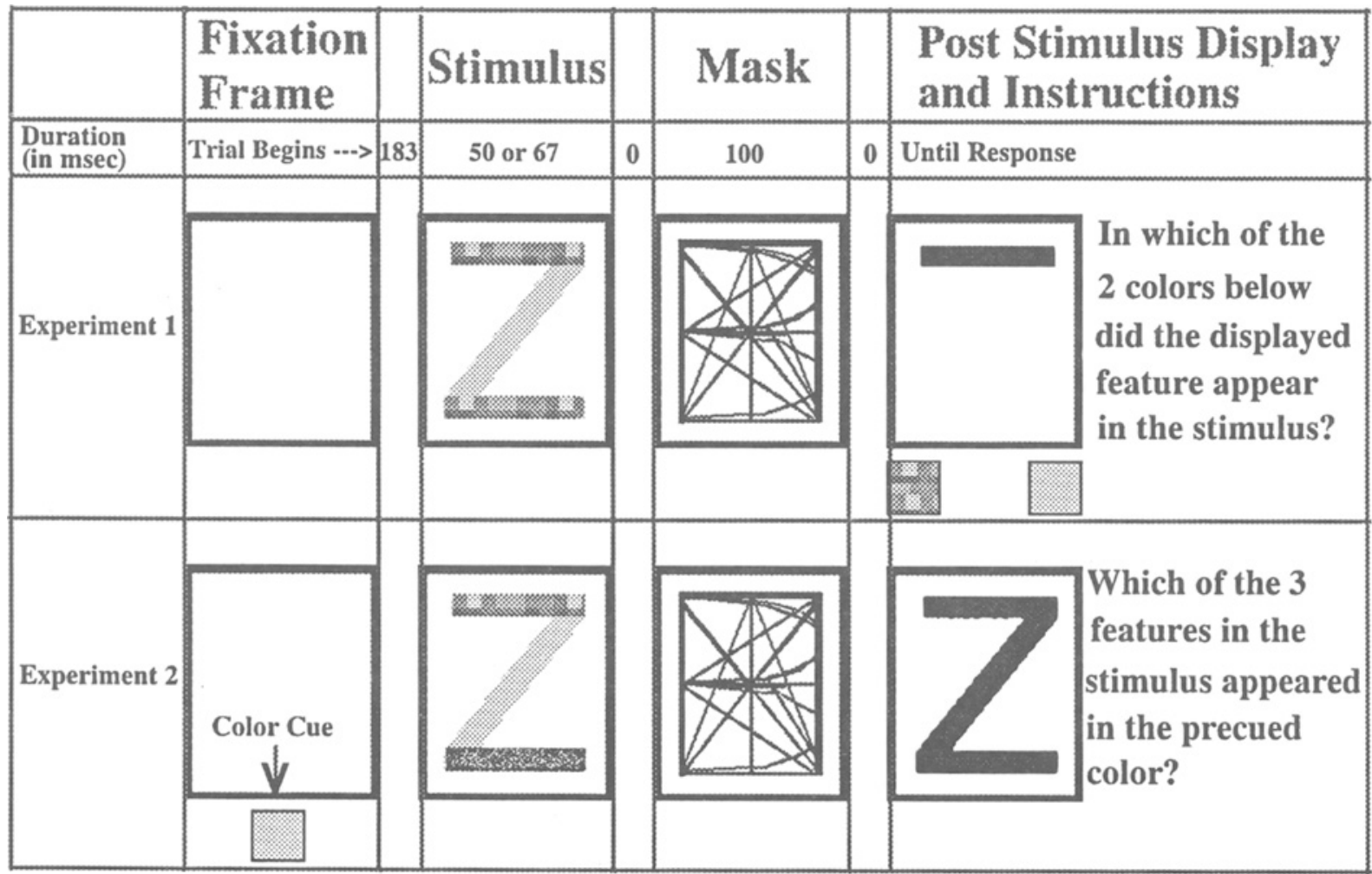

Figure 5. Summary of trial sequences in Experiments 1 and 2.

formance expressed in mean proportion correct as well as adjusted item scores (i.e., with feature variance removed). Given that familiarity and the R\&R measure of figural goodness were correlated within the stimulus set $[R(28)=-.649, p<.001]$, partial correlations are also reported. The partial correlations are between each stimulus property (i.e., familiarity or figural goodness) and item performance, with the other stimulus property controlled.

As can be seen in Table 2, familiarity ratings correlated significantly with item performance. This was true for both simple and partial correlations as well as for both types of item-performance measures (i.e., proportion correct and adjusted scores). In contrast, the $R \& R$ measure of figural goodness correlated significantly only with adjusted item-performance scores. However, after partialing out the effects of familiarity, the correlation between $R \& R$ values and adjusted item scores was no longer significant, suggesting that the simple correlation between these variables is spurious.

The results of the subject and item analysis reflect a very consistent pattern of findings, indicating a lettercontext-superiority effect in the color-identification task. The size of the effect, about $4 \%$, was far smaller than the LCE reported by Schendel and Shaw (1976). However, in contrast to the effect reported by Schendel and Shaw, the present effect cannot be explained by structural redundancy. As discussed previously, the subjects' knowl- edge of the structural redundancies of letters was noninformative in the color-identification task employed in the present experiment. In addition, figural goodness, as measured by Garner's R\&R index, did not have a significant effect and thus cannot account for the observed lettersuperiority effect (LSE).

\section{EXPERIMENT 2}

Experiment 1 demonstrated a letter-context-superiority effect that is not attributable to either structural redundancy or figural goodness. However, before it can be concluded that this context-superiority effect is truly a per-

Table 1

Mean Proportion Correct by Stimulus Type in Experiments 1 and 2

\begin{tabular}{lllll}
\hline & \multicolumn{5}{c}{ Experiment } \\
\cline { 2 - 6 } \cline { 5 - 6 } \multicolumn{1}{c}{ Stimulus Type } & $M$ & $S E$ & $M$ & $S E$ \\
\cline { 2 - 6 } & .800 & .024 & .822 & .018 \\
Letters & & & & \\
High-figural-goodness & .760 & .027 & .780 & .024 \\
nonletters & & & & \\
Low-figural-goodness & .754 & .025 & .776 & .019 \\
nonletters & & & & \\
Mean & .771 & .025 & .793 & .020 \\
\hline
\end{tabular}


Table 2

Simple and Partial Correlations Between Item Performance and R\&R Values and Between Item Performance and Familiarity Ratings in Experiments 1 and 2

\begin{tabular}{|c|c|c|c|c|c|}
\hline \multirow[b]{2}{*}{ Correlation } & \multirow{2}{*}{$\begin{array}{c}\text { Item } \\
\text { Performance }\end{array}$} & \multicolumn{2}{|c|}{ R\&R Values } & \multicolumn{2}{|c|}{ Familiarity Ratings } \\
\hline & & Experiment 1 & Experiment 2 & Experiment 1 & Experiment 2 \\
\hline \multirow[t]{2}{*}{ Simple } & Proportion correct & -.20 & -.26 & $.56 \dagger$ & $.46^{*}$ \\
\hline & Adjusted score & $-.38^{*}$ & -.37 & $.71 \dagger$ & $.53 \dagger$ \\
\hline \multirow[t]{2}{*}{ Partial } & Proportion correct & .27 & .05 & $.58 \S$ & $.48 \ddagger$ \\
\hline & Adjusted score & .14 & -.04 & $.65 \$$ & $.41 \neq$ \\
\hline
\end{tabular}

Note-Adjusted performance scores were obtained by removing feature variance (see text for exact procedure). Partial correlations are between measures of one stimulus property (e.g., $R \& R$ measure of figural goodness) and item performance, with the measures of the other stimulus property (e.g., familiarity) controlled. Critical values for the simple correlation coefficients: ${ }^{*} r(28)=.375$,

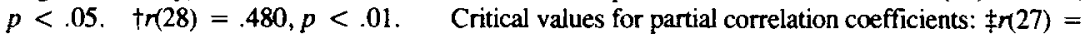
$.382, p<.05 . \quad \S r(27)=.488, p<.01$

ceptual rather than a postperceptual effect, another alternative explanation requires consideration. Simply put, the LSE demonstrated in Experiment 1 may reflect a memory rather than a perceptual advantage of the letter versus the nonletter contexts (see Johnston, 1981; McClelland \& Rumelhart, 1981; Pomerantz et al., 1977). A memory interpretation of visual-context effects does not assume any quantitative or qualitative differences in the perceptual processing of familiar versus nonfamiliar contexts. Rather, it is hypothesized that the perceptual information extracted from the analysis of familiar stimuli is more durable (i.e., less susceptible to forgetting) because it can be retained as one unit and its components can later be specified from memory.

One strategy for testing a perceptual versus a memory interpretation of visual-context effects is to minimize the memory component involved in the experimental task. For example, in the study of the WSE, the replacement of the whole-report procedure by the partial-report procedure resulted in a substantial decrease in the memory component of the letter-identification task (Reicher, 1969). As pointed out by McClelland and Rumelhart (1981) with regard to the WLE: "it is not reasonable to argue that the effect is due to forgetting letters that have been perceived. It is hard to see how a single letter once perceived, could be subject to a greater forgetting than a letter in a word" (p. 376).

Nevertheless, whether or not the WSE or the WLE is attributable, at least in part, to top-down perceptual processing is still highly controversial in current theories of word perception. The interactive-activation model proposed by McClelland and Rumelhart (1981) accounts for context effects in word perception by postulating top-down as well as bottom-up perceptual processing. In contrast, the hierarchical model suggested by Johnston and McClelland (1980) is strictly a bottom-up account of word perception and provides a memory explanation of context effects.

It is important to emphasize that memory and perceptual accounts of visual-context effects are not necessarily mutually exclusive. As pointed out by Krueger (1975), it may be "that familiarity can affect either memory or perception, depending on which is more critical to overall task performance" (p. 954). In fact, memory effects in letter identification have been convincingly demonstrated (Baddeley, 1964). Furthermore, logically it is necessary that any effects of familiarity on perception involve access to some sort of memory representation. What is not clear, however, is whether or not in addition to memory-based advantages, visual-context effects also represent an example of top-down perceptual processing.

Experiment 2 was designed to explore this issue with regard to the context effect demonstrated in Experiment 1. The strategy employed in the present study is similar to the one used by Mermelstein, Banks, and Prinzmetal (1979). These investigators varied the memory requirements of an FSE-like task by contrasting a visual-search condition to a memory-search condition. The former involved the presentation of a target feature (e.g., eyes, nose, etc.) prior to presentation of the stimulus (i.e., face or nonface), whereas the latter involved a poststimulus display of the target feature. It was assumed that the visual-search condition minimized the memory component involved in the task relative to the memory-search condition. Mermelstein et al. found that features were identified better in a face context relative to a scrambledface context in the memory-search condition, whereas the reverse was true in the visual-search condition. Mermelstein et al. (1979) concluded that "in a task that depends on memory for configuration, having the array configured as a good form helps detection of a constituent part, but in a task that requires perceptual analysis of the configuration, a good form hinders detection of a constituent part"' (p. 472). These investigators further argued that their conclusions apply beyond face stimuli.

The present experiment employed a visual-search version of the task used in Experiment 1. On each trial, subjects were instructed to scan the stimulus display for a feature in a preassigned color. The colors red, green, and yellow were used. Each of the alternative colors on any given trial specified one, and only one, feature. If Mermelstein et al. (1979) were correct, one would expect the 
effect found in Experiment 1 to be reversed, eliminated, or at least reduced under the conditions used in Experiment 2.

\section{Method}

Subjects. Twenty undergraduate students at the University of Waterloo participated in the experiment. None of the subjects had participated in Experiment 1. All subjects had normal or correctedto-normal vision, and each subject was paid \$10 following completion of the experimental session.

Procedure. The method for this experiment was the same as in Experiment 1 except for the following changes. As summarized in the bottom half of Figure 5, the major difference concerned the presentation sequence in each trial. Prior to stimulus presentation, the subjects were informed of the color of the target feature by the presentation of a rectangular color patch directly below the fixation frame. The color patch remained on until the trial was initiated. Once a trial was initiated, $183 \mathrm{msec}$ elapsed before the onset of the colored stimulus. Following the offset of the stimulus display, a white feature mask was presented for $100 \mathrm{msec}$. Following the offset of the mask, the stimulus was again presented, but this time in white. The subjects were required to select the feature that appeared in the precued color. Each subject indicated his/her decision by pointing an arrow to the selected feature using a mouse and pressing the left button on the mouse. The selected feature was then colored blue. The subject could change his/her selection of a feature until he/she felt he/she had the correct choice. The subject's choice was then recorded, and the next trial sequence began.

Another difference between Experiment 2 and Experiment 1 was in terms of the color alternatives employed. Yellow was added to the red and green color alternatives employed in Experiment 1. All three colors were used in each trial, resulting in a one-to-one correspondence between colors and features. However, as in Experiment 1 , there were six possible feature-color mappings. Consequently, there was no difference between experiments in terms of the randomization of practice and experimental trials. Finally, the stimulus-duration values assigned to the subjects on the basis of their performance during practice trials were also identical to those assigned in Experiment 1: 16 subjects received a stimulus duration of $50 \mathrm{msec}$, and the remaining 4 received a stimulus duration of $67 \mathrm{msec}$.

\section{Results and Discussion}

In general, the results of Experiment 2 were very similar to the findings of Experiment 1. As can be seen in Table 1, stimulus type had a significant effect on performance $[F(2,38)=11.09, p<.001]$. More specifically, performance with letter contexts was significantly better relative to performance with both high-figural-goodness nonletters $[t(19)=3.52, p<.01]$ and low-figuralgoodness nonletters $[t(19)=5.51, p<.001]$. The difference across the two nonletter sets was not significant $[t(19)=.38, p=.71]$.

Item analysis. Mean proportion correct, $R \& R$ values, and mean familiarity ratings for each of the stimuli employed in the present experiment are shown in Figures 2,3 , and 4 . Table 2 presents the simple and partial correlations between item performance and the $R \& R$ measure of figural goodness and between item performance and familiarity ratings. A comparison of these correlations in Experiments 1 and 2 reveals that although the absolute values of the correlations tended to be somewhat lower in Experiment 2 than in Experiment 1, the pattern of the correlations was remarkably similar across exper- iments. As in Experiment 1, familiarity ratings, but not $R \& R$ values, were significantly correlated with item performance.

Because of the importance of contrasting the visualsearch condition used in the present experiment with the memory-search condition employed in Experiment 1, a $2 \times 3$ analysis of variance that evaluated experiment (i.e., Experiment 1 vs. Experiment 2) as a between-subject factor and stimulus type (i.e., letters vs. high-figuralgoodness nonletters vs. low-figural-goodness nonletters) as a within-subject factor was performed. As is obvious from inspection of Table 1, the interaction between experiment and stimulus type was not significant $(F<1)$.

At first glance, the present results may seem at odds with those of a number of researchers who have reported inferiority effects associated with visual search for a stimulus attribute in configurations with high figural goodness (e.g., Banks \& Prinzmetal, 1976; Mermelstein et al., 1979; Prinzmetal \& Banks, 1977). However, there are a number of differences between our paradigm and earlier ones that make the discrepancy more apparent than real. We discuss one such difference here to highlight the difficulties involved in generalizing from these previous studies to the present one. One difference between our paradigm and those of previous investigators is that our subjects were required to search a display for the presence of a particular color and to identify the pattern segment in that color. In contrast, previous studies reporting inferiority effects have required subjects to search displays for a pattern attribute within good or bad patterns. One important difference is that stimulus selection, in our paradigm, takes place using a stimulus dimension other than the one used to produce the goodness/badness of the configuration. Because color and shape (or pattern) are separable dimensions, stimulus selection based on color information would be expected to proceed without strong influence from the shape/pattern properties of the display (see also Egeth, Virzi, \& Garbart, 1984). In contrast, in studies reporting "camouflaging" effects of good configurations, search was carried out for patterns among patterns, that is, along the same dimension used to define the goodness/badness of the configuration (e.g., Banks \& Prinzmetal, 1976).

Furthermore, consider the possibility that the locus of the effects of familiarity and of figural goodness are at different stages of visual-information processing. We would expect that our facilitatory effects of familiarity take place at a relatively early stage involved in the construction of a perceptual representation of the stimulus. In contrast, the camouflaging effects reported probably take place at a later stage in which this representation (or the display itself) is searched for a particular piece of information. We strongly suspect that the perceptually enhancing effects of familiarity that we found in the present study were operating in previous studies demonstrating inferiority effects. However, these early effects were camouflaged by even stronger effects going in the opposite direction in later stages involved in searching the representation or display along the pattern/shape dimension. 
Therefore, we do not dispute the findings of earlier reports demonstrating inferiority effects. Instead, we argue that our paradigm, in contrast with earlier ones, was able to reveal the facilitatory effects of familiarity on perceptibility without the camouflaging effects described earlier.

Similarly, a potentially troublesome apparent discrepancy between our results and those of Prinzmetal and Millis-Wright (1984) can probably also be attributed to subtle but important procedural differences between our experiments and theirs. They presented subjects with three-letter words and nonwords in which the letters were in various colors. The task was to search the letter string for one of two letters and to report the letter and the color in which it was presented (in some experiments, subjects searched the string for a single letter and reported the color of that letter). They found that subjects were more likely to report the color of another letter for words than for nonwords. That is, there were more feature-conjunction errors for words than for nonwords, a word-inferiority effect in a color-perception task. Why did we not find a similar letter-inferiority effect? The answer may reside in the fact that the Prinzmetal and Millis-Wright paradigm, as other studies that have shown inferiority effects, required subjects to actively search the display for a particular pattern and then to report an attribute of that pattern. This search activity appears to have made the task quite difficult, as evidenced by the relatively long exposure durations reported by Prinzmetal and Millis-Wright (168 msec, on average, across their five experiments). Again, it seems possible that the locus of this inferiority effect is in a stage of processing that is subsequent to the construction of a perceptual representation of the stimulus. In any case, it is clear that there are a number of procedural differences that make comparisons across their paradigm and ours rather difficult (e.g., longer exposure durations, search for patterns among patterns, etc.).

Thus, in contrast to the implications of Mermelstein et al.'s (1979) position, in the present experiment, neither stimulus familiarity nor figural goodness have impaired performance in a visual-search task. In fact, the findings of Experiment 2 replicated almost exactly those of Experiment 1 . Clearly, further research is required in order to isolate the conditions in which stimulus familiarity and/or figural goodness hinder the identification of constituent elements. Finally, to the extent that the visualsearch condition employed in the present experiment was successful in reducing the memory requirements of the color-identification task, an account of the LSE demonstrated in this experiment solely in terms of a memory advantage is rendered less plausible.

\section{GENERAL DISCUSSION}

The validity of the interpretation of the letter-context effects demonstrated in the present experiments as perceptual in nature rests entirely on the adequacy of the controls provided in these experiments for ruling out postperceptual accounts. Accordingly, with regard to the implications of the present results, three issues should be discussed. First, we evaluate the basis for rejecting structural redundancy, figural goodness, and memory advantage as satisfactory explanations of the present findings. Second, a top-down perceptual processing account of the reported context effects is outlined. Third, some of the potential contributions of the color-judgment paradigm introduced in the present study are discussed.

In attempting to interpret the context effects reported here, it is crucial to evaluate the logical status of the inferences permitted by the present methodology. In this section, the case against each one of the three alternative explanations, namely, structural redundancy, figural goodness, and memory advantage, is outlined.

\section{Structural Redundancy}

With respect to structural redundancy, we adopt a very strong position. More specifically, we propose that the methodology employed here logically excluded structural redundancy as a possible explanation of the present results. Consider, for example, the results of Experiment 1. In this experiment, the subjects identified the color of a letter fragment more accurately in letter than in nonletter contexts. Note that this letter-superiority effect was demonstrated under experimental conditions that excluded any possible influence of structural redundancy. This is the case because the identification of any given letter could only have been used to infer its parts, not their color. In other words, the subjects' knowledge of the structural redundancy in letters was rendered noninformative in the color-judgment task.

\section{Figural Goodness}

In the present experiments, figural goodness did not correlate with performance. Our results are consistent with those of Pomerantz et al. (1977), which demonstrated that symmetry per se did not produce configural-superiority effects. However, our case against figural goodness is largely dependent on the adequacy of the $R \& R$ index (Garner, 1962, 1974) as a measure of this construct. Further studies employing alternative measures of figural goodness (e.g., Palmer, 1977) are required in order to assess the generality of the present findings. Nevertheless, we feel moderately confident in ruling out figural goodness as a satisfactory explanation of the present findings.

\section{Memory versus Perception}

The strategy we adopted in attempting to decide between a perceptual versus a memory account of the present findings was patterned after Mermelstein et al.'s (1979) approach of contrasting a visual-search condition with a memory-search condition. As mentioned earlier, the basic assumption underlying this approach is that there is a substantial reduction in the requirement for memory processing in visual search relative to memory search. However, given that we do not have an independent means of measuring the memory component of a task, such manipula- 
tions may not lead to a conclusive resolution of this issue. Thus, a memory account of the present results, although less plausible, remains a logical possibility.

Keeping in mind the necessity for caution, it may still be useful to consider one potential top-down perceptual account of the present results. More specifically, the interactive-activation model (McClelland \& Rumelhart, 1981; Rumelhart \& McClelland, 1982) provides a potentially valuable framework for the interpretation of lettercontext effects. This model postulates three levels of detectors in word perception: feature level, letter level, and word level. Given that the original exposition of this model was aimed at explaining visual-context effects in word perception, only the assumptions concerning the interaction (i.e., excitation and/or inhibition) between the word and letter levels were fully specified. Briefly, according to this model, letter-level detectors excite consistent word-level detectors, which in turn feed back to the letter level, strengthening the activation of constituent letters and thereby increasing their perceptibility. However, if similar assumptions are made with regard to the interaction between the letter and feature levels, then the extended model clearly predicts that features would be more perceptible in letter than in nonletter contexts. Note that the present implementation of the model does not include feedback from the letter level to the feature level.

The interactive-activation model extended in such a way can easily account for the findings of Experiments 1 and 2. More specifically, performance in the color-judgment task requires accurate perceptual integration of shape and color information. Under degraded stimulus presentation, the more efficient processing of features in letter versus nonletter contexts may allow for better integration of feature and color information in the former relative to the latter condition. Obviously, it is premature to attempt to specify the mechanisms that may underlie the lettercontext effects demonstrated in the present experiments. Consequently, the potential relevance of the interactiveactivation framework to the interpretation of the present findings is yet to be established.

Finally, as pointed out by Purcell and Stewart (1988), "the number of accepted context effects now requires a short list of trigrams just to keep them from becoming confused with each other"' (p. 355). Although these effects are often mentioned in conjunction with one another, a systematic attempt at integrating and contrasting the various findings has yet to be achieved. An important prerequisite for such an enterprise is the employment of experimental paradigms that can be applied to the study of visual-context effects across a wide variety of stimulus materials. It is precisely for this reason that the proposed paradigm, as well as other approaches that can be broadly applied, such as the face-detection-effect paradigm (e.g., Purcell \& Stewart, 1981, 1985, 1986, 1988; Purcell, Stewart, Botwin, \& Kreigh, 1983), may prove valuable. Given the similarities in the theoretical questions investigated by researchers of various visual-context effects, the development of a unitary conceptual-methodological framework has distinct advantages.

\section{REFERENCES}

ATTNEAVE, F. (1959). Applications of information theory to psychology. New York: Holt.

BADDELEY, A. D. (1964). Immediate memory and the "perception" of letter sequences. Quarterly Journal of Experimental Psychology, 16, 364-367.

Banks, W. P., \& Prinzmetal, W. (1976). Configurational effects in visual information processing. Perception \& Psychophysics, 19, 361-367.

Baron, J. (1978). The word-superiority effect: Perceptual learning from reading. In W. K. Estes (Ed.), Handbook of learning and cognitive processes (Vol. 6, pp. 131-166). Hillsdale, NJ: Erlbaum.

Biederman, I. (1972). Perceiving real world scenes. Science, 177, 77-80.

Biederman, I. (1981). On the semantics of a glance at a scene. In M. Kubovy \& I. R. Pomerantz (Eds.), Percepual organization (pp. 213-253). Hillsdale, NJ: Erlbaum.

Biederman, I., Glass, A. L., \& Stacey, E. W., Jr. (1973). Scanning for objects in real world scenes. Journal of Experimental Psychology, 97, 22-27.

Chen, L. (1982). Topological structure in visual perception. Science, 218, 699-700.

EARHARD, B. (1980). The line-in-object superiority effect in perception: It depends on where you fix your eyes and what is located at the point of fixation. Perception \& Psychophysics, 28, 9-18.

Earhard, B. \& ARMit AGE, R. (1980). From an object-superiority effect to an object-inferiority effect with movement of the fixation point. Perception \& Psychophysics, 28, 369-376.

Egeth, H., Virzi, R. A., \& Garbart, H. (1984). Searching for conjunctively defined targets. Joumal of Experimental Psychology: Human Perception \& Performance, 10, 32-39.

EnNs, J. T., \& Gilani, A. B. (1988). Three-dimensionality and discriminability in the object-superiority effect. Perception \& Psychophysics, 44, 243-256.

EnNS, J. T., Prinzmetal, W. (1984). The role of redundancy in the object-line effect. Perception \& Psychophysics, 35, 22-32.

GARNER, W. R. (1962). Uncertainty and structure as psychological concepts. New York: Wiley.

GARNER, W. R. (1974). The processing of information and structure. Potomac, MD: Erlbaum.

Garner, W. R., \& Clement, E. D. (1963). Goodness of pattern and pattern uncertainty. Journal of Verbal Learning \& Verbal Behavior, 2, 446-452.

GoTTWALD, R. L., \& GARNER, W. R. (1972). Effects of focusing strategy on speeded classification with grouping, filtering, and condensation tasks. Perception \& Psychophysics, 11, 179-182.

Gyoba, J., Arimura, M., \& Maruyama, K. (1980). Visual identification of line segments embedded in human face patterns. Tohoku Psychologica Folia, 39, 113-120.

HANDEL, S., \& ImaI, S. (1972). The free classification of analyzable and unanalyzable stimuli. Perception \& Psychophysics, 12, 108-116.

Homa, D., Haver, B., Schwartz, T. (1976). Perceptibility of schematic face stimuli: Evidence for a perceptual Gestalt. Memory \& Cognition, 4, 176-185.

JoHnston, J. C. (1974). The role of contextual constraint in the perception of letters in words. Unpublished doctoral dissertation, University of Pennsylvania, Philadelphia.

JoHNSTON, J. C. (1978). A test of the sophisticated guessing theory of word perception. Cognitive Psychology, 10, 123-153.

JoHNSTON, J. C. (1981). Understanding word perception: Clues from studying the word-superiority effect. In $\mathbf{O}$. J. L. Tzeng \& $\mathrm{H}$. Singer (Eds.), Perception of print: Reading research in experimental psychology (pp. 65-84). Hillsdale, NJ: Erlbaum.

Johnston, J. C., \& MCClelland, J. L. (1980). Experimental tests of a hierarchical model of word identification. Joumal of Verbal Leaming \& Verbal Behavior, 19, 503-524.

KinCHLA, R. A. (1977). The role of structural redundancy in the perception of visual targets. Perception \& Psychophysics, 22, 19-30.

KLEIN, R. (1978). Visual detection of line segments: Two exceptions to the object superiority effect. Perception \& Psychophysics, 24, 237-242. 
Krueger, L. E. (1975). Familiarity effects in visual information processing. Psychological Bulletin, 82, 949-974.

Lanze, M., Maguirf, W., \& Weisstein, N. (1985). Emergent features: A new factor in the object-superiority effect? Perception \& Psychophysics, 38, 438-442.

Lanze, M., Weisstein, N., \& Harris, J. R. (1982). Perceived depth vs. structural relevance in the object-superiority effect. Perception \& Psychophysics, 31, 376-382.

Massaro, D. W. (1973). Perception of letters, words, and nonwords. Journal of Experimental Psychology, 100, 349-353.

MCCLELLAND, J. L. (1978). Perception and masking of wholes and parts. Joumal of Experimental Psychology: Human Perception \& Performance, 4, 210-223.

McClelland, J. L., \& Miller, J. (1979). Structural factors in figure perception. Perception \& Psychophysics, 26, 221-229.

MCClelland, J. L., \& Rumelhart, D. E. (1981). An interactive activation model of context effects in letter perception: Part 1. An account of basic findings. Psychological Review, 88, 375-407.

Mermelstein, R., Banks, W., Prinzmetal, W. (1979). Figural goodness effects in perception and memory. Perception \& Psychophysics, 26, 472-480.

NisSEN, M. J. (1979, November). Identification, localization, and the processing of conjunctions. Paper presented at the meeting of the Psychonomic Society, Phoenix, AZ.

Palmer, S. E. (1975). The effects of contextual scenes on the identification of objects. Memory \& Cognition, 3, 519-526.

PALMER, S. E. (1977), Hierarchical structure in perceptual representation. Cognitive Psychology, 9, 441-474.

Pomerantz, J. R. (1981). Perceptual organization in information processing. In M. Kubovy \& J. R. Pomerantz (Eds.), Perceptual organization (pp. 141-180). Hillsdale, NJ: Erlbaum.

Pomerantz, J. R., Sager, C. S., \& Stoever, R. J. (1977). Perception of wholes and of their component parts: Some configural superiority effects. Journal of Experimental Psychology: Human Perception \& Performance, 3, 422-435.

Prinzmetal, W., \& BANKs, W. P. (1977). Good continuation affects visual detection. Perception \& Psychophysics, 21, 389-395.

Prinzmetal, W., \& Millis-Wright, M. (1984). Cognitive and linguistic factors affect visual feature integration. Cognitive Psychology, 16, 305-340.

Prinzmetal, W., Presti, D. E., \& Posner, M. I. (1986). Does attention affect visual feature integration? Journal of Experimental Psychology: Human Perception \& Performance, 3, 361-369.
Purcell, D. G., Stewart, A. L. (1981). A face superiority effect. Bulletin of the Psychonomic Society, 18, 73.

Purcell, D. G., \& Stewart, A. L. (1985). The face detection effect. Bulletin of the Psychonomic Society, 23, 288.

Purcell, D. G., \& STEWART, A. L. (1986). The face-detection effect. Bulletin of the Psychonomic Society, 24, 118-120.

Purcell, D. G., \& Stewart, A. L. (1988). The face-detection effect: Configuration enhances detection. Perception \& Psychophysics, 43, 355-366.

Purcell, D. G., Stewart, A. L., Botwin, M., \& Kreigh, R. (1983). A face superiority effect: Hemiretina effects. Bulletin of the Psychonomic Society, 21, 351.

ReICHER, G. M. (1969). Perceptual recognition as a function of meaning fulness of stimulus material. Journal of Experimental Psychology, 81, 275-280.

RumelharT, D. E., \& MCClelland, J. L. (1982). An interactive activation model of context effects in letter perception: Part 2. The contextual enhancement effect and some tests and extensions of the model. Psychological Review, 89, 60-94.

Schendel, J. D., \& Shaw, P. (1976). A test of the generality of the word-context effect. Perception \& Psychophysics, 19, 383-393.

Treisman, A., Gelade, G. (1980). A feature integration theory of attention. Cognitive Psychology, 12, 97-136.

Treisman, A., \& Schmidt, H. (1982). Illusory conjunctions in the perception of objects. Cognitive Psychology, 14, 107-141.

VAN SANTEN, J. P. H., \& Jonides, J. (1978). A replication of the facesuperiority effect. Bulletin of the Psychonomic Society, 12, 378-380.

WeISSTEIN, N., \& HARRIS, C. S. (1974). Visual detection of line segments: An object-superiority effect. Science, 186, 752-755.

Weisstein, N., Willuams, M. C., \& HarRis, C. S. (1982). Depth, connectedness, and structural relevance in the object-superiority effect: Line segments are harder to see in flatter patterns. Perception, 11, 5-17.

Williams, A., \& Weisstein, N. (1978). Line segments are perceived better in coherent context than alone: An object-line effect in visual perception. Memory \& Cognition, 6, 85-90.

WOMERSLEY, M. (1977). A contextual effect in feature detection with application of signal detection methodology. Perception \& Psychophysics, 21, 88-92.

(Manuscript received October 18, 1989; revision accepted for publication July 24,1992 .) 